\title{
Valores, creencias y representaciones sociales de los motociclistas infractores de la ciudad de Neiva ${ }^{1}$
}

\author{
Miguel Ángel Mahecha Bermúdez \\ Universidad Surcolombiana, Colombia \\ miguelangel.mahecha@usco.edu.co \\ Dagoberto Páramo Morales \\ Universidad del Norte, Colombia \\ dparamo@uninorte.edu.co \\ Elías Ramírez Plazas \\ Universidad Surcolombiana, Colombia \\ elramirez2008@gmail.com
}

\section{Resumen}

Este artículo analiza la cultura infractora mantenida por los motociclistas de la ciudad de Neiva, la cual justifica y estimula el actuar infractor. El análisis emplea las contribuciones teóricas del modelo de cultura de Hofstede (2001) y las complementa con otros estudios que se centran en las representaciones sociales y sus patrones culturales, esto es lo etnometodológico y el interaccionismo simbólico. Los hallazgos señalan que los instrumentos tienen validez de contenido, pragmática, concurrentes, predictiva, y que hay una fuerte conexión entre la cultura, las infracciones y la accidentalidad. Que la escasa importancia dada a la seguridad vial y al acatamiento normativo es el fruto de un permisivo actuar de la autoridad y de las escuelas de tránsito en la regulación de la seguridad y movilidad vial de la ciudad.

Palabras clave: Comportamiento del consumidor, valores, creencias, representaciones sociales, motociclistas infractores.

\section{Values, beliefs and social representations of motorcyclists in violation of the city of Neiva}

\section{Abstract}

This paper analyzes the maintained infringing culture of motorcyclists in Neiva which justifies and encourages the offending act. The analysis uses the theoretical contributions of Hofstede's culture model (2001) and they're supplemented by other studies focused on social representations and their cultural patterns. That is to say the ethnomethodology and the symbolic interaction. The findings indicate that the instruments have content validity, pragmatic, concurrent, predictive, and

1 El presente proyecto fue registrado y financiado por la Vicerrectoría de Investigaciones y Proyección Social de la Universidad Surcolombiana con el nombre de "Configuración de las representaciones mentales de los motociclistas que han infringido las normas de tránsito en Neiva". 
that there is a strong connection between culture, offenses and accidents. That the low importance given to road safety and regulatory compliance is the result of a permissive act of authority and traffic schools in regulating safety and road mobility in the city.

Keywords: Consumer behavior, values, beliefs, social representations, motorcyclist offenders.

\section{Introducción}

Las recurrentes infracciones de los conductores, especialmente de los motociclistas, ocupan un lugar importante en las cifras de accidentalidad: 1.2 millones de personas perecen y 50 millones quedan con lesiones a nivel mundial (Bachoo, Bhagwanjee \& Govender, 2013); en Colombia, 6.219 casos de muerte se registraron en el año 2013 (Vargas, 2013) y en Neiva, 8 muertes y 116 lesionados (Córdoba Mazabel \& Riaño Angarita, 2013). Esta conducta se debe a una preocupación por el cumpliento de actividades laborales, familiares y escolares (Xie \& Parker, 2002; Cantin, Lavallière, Simoneau \& Teasdale, 2009; Naveteur, Coeugnet, Charron, Dorn, Anceaux y fançoise, 2013); a experiencias desagradables (Olea, Nordfjærnb \& Rundmoc, 2012); a un deseo de autorealización por razones de género (Bachoo, Bhagwanjee \& Govender, 2013; Ruiz, Gómez, Beltrán, Lamus \& Leal, 2014; Poncela, 2012); a una inadecuada vigilancia de las escuelas de conducción y las autoridades de tránsito (Ozkan, Lajunen, Chliaoutakis, Parker \& Summala, 2006; Nallet, Bernard \& Chiron, 2008; González \& Ossa, 2011); al estilo de conducción (Walker, Stantonb \& Salmonc, 2011) y a los valores culturales (Gayg1sız, 2010; Gomes, 2013).

La revisión teórica sobre infracciones de los conductores de motociclistas indican que no existen estudios sobre valores, creencias y representaciones sociales; por esta razón, el presente artículo pretende contribuir en estos aspectos y resaltar la importancia de conocer la cultura de los infractores de la ciudad de Neiva ya que esta define las formas de pensar, sentir, actuar e interactuar de las personas, las instituciones y las posesiones, sin descuidar la manera como dicha cultura se fortalece y comparte por sus miembros.

\section{Referente conceptual}

El creciente número de infracciones y su relación con los accidentes, según Xie \& Parker (2002) se debe a los inconvenientes que tiene el conductor a menudo en su movilidad como congestión del tráfico, conversaciones imprevistas entre los mismos conductores o un conductor y un peatón, vehículo averiado, entre otras. Lo anterior hace que el tiempo dispuesto para el desarrollo de los compromisos, sean estos laborales, familiares o personales, esté condicionado $\mathrm{y}$, por tanto, genera impaciencia y estrés (Naveteur, Coeugnet, Charron, Dorn, Anceaux y fançoise, 2013). Esta impaciencia y estrés provoca, señalan Cantin, Lavallière, Simoneau \& Teasdale, (2009), que los conductores reprochen todo tipo de obstrucción que afecte su propia movilidad y no toleren cuestionamiento cuando cometen la infracción.

Olea, Nordfjærnb \& Rundmoc (2012) señalan que las malas experiencias relacionadas con robo, amenazas, entre otras, paulatinamente son reflejadas en los hábitos y estilos de conducción cuando se encuentran en situaciones equivalentes, lo que produce una actuación impulsiva. Contraria a esta posición, Bachoo, Bhagwanjee \& Govender (2013) y Poncela (2012) señalan que la participación de los hombres jóvenes en el aumento de las infracciones obedece a la concepción de "superioridad" entre los sexos. De ahí que, las cualidades de condución estén relacionadas con situaciones que destaquen al hombre. Algunas situaciones son manejar con exceso de velocidad, bajo estado de embriguez, maniobrar ante fallas de la infraestructura víal (Gayg1s1z, 2010; Gomes, 2013) o a través de la violencia a otros conductores (Ruiz, Gómez, Beltrán, Lamus \& Leal, 2014). Una opinión diferente tiene Ozkan, Lajunen, Chliaoutakis, Parker \& Summala (2006) cuando mencionan que 
los estilos de conducción inapropiados se deben a la falta de una adecuada regulación de las escuelas de conducción para expedir la licencia, a saber: un examen teórico y médico general sin profundizar en aspectos psicologicos como ansiedad, agresividad, impulsividad, miedos. Esto lleva a que los infractores, por omisión del control de las autoridades de tránsito (González \& Ossa, 2011), a no tener percepción de peligro y sanción (Nallet, Bernard \& Chiron, 2008).

Walker, Stantonb \& Salmonc (2011), en cambio, sostiene que los beneficios de la moto en comparación con los de un auto da lugar a que las infracciones sean más recurrentes; el tamaño, la rápidez y una mejor panoramica de la vía son aspectos que permite un estilo de conducción versatil y controlado. A diferencia de los motociclistas, el tamaño y la coordinación son aspectos que requieren demasiada atención para los conductores de autos, por tanto, limitan la tendencia a las infracciones. Finalmente, Gaygisız (2010) retoma los valores de la cultura, aduciendo que aquellos definen el desempeño de las instituciones, particulamente las encargadas de la seguridad y movilidad vial; desafortunamente, el número de fatalidades por accidentes de tránsito y su incremento señalan la poca importancia de este aspecto en la conducta infractora.

\section{Metodología}

El estudio recurrió a dos tipos de investigaciones: exploratoria-descriptiva y correlacional. La primera se usó para identificar los valores, las creencias y las representaciones mentales de los motociclistas que infringen las normas de tránsito en Neiva y la segunda, para explicar su relación con el comportamiento infractor y la accidentalidad. La investigación exploratoria-descriptiva estuvo guiada teóricamente por el modelo cultural (Schein, 1985) el cual sostiene que la cultura se explica mediante sus tres componentes esenciales: valores, creencias y representaciones mentales. La recolección de la información exploratoriadescriptiva utilizó las contribuciones de Strauss \& Corbin (1998) y Garfinkel (1967) y como métodos la observación directa, la observación participante, la entrevista a profundidad, registro fílmico, registro fotográfico, registro de notas y panel de expertos. Para el tamaño de la muestra se utilizó la saturación teórica (50 entrevistas a profundidad) y cubrieron todos los estratos. Una vez realizadas las entrevistas a profundidad, se clasificó y se codificó la información con base en los marcos teóricos sobre valores, creencias y representación sociales (dimensiones y variables).

Los hallazgos de la investigación cualitativa de valores, las creencias y las representaciones sociales, fueron la base para diseñar cuestionario estructurado (investigación descriptiva) en escala Likert (Desacuerdo 122345 Acuerdo) y de respuestas múltiples. El cuestionario fue sometido a sesiones de grupo con quince (15) motociclistasinfractores de tránsito queno sabían leer, para constatar la claridad de las preguntas. Después de hacer los ajustes correspondientes, se aplicó el instrumento final a 400 motociclistas infractores de tránsito en Neiva (margen de confianza del $95 \%$ y un error máximo admitido en las estimaciones del 4.7\%). La selección de la muestra se hizo de manera aleatoria. El análisis de datos cuantitativos (correlacional) se hizo mediante el procesamiento de 380 encuestas utilizando el paquete estadístico SPSS, el cual permitió confirmar que los instrumentos aplicados tenían fiabilidad y validez.

\section{Resultados}

Fiabilidad y validez de instrumentos. Los resultados del alpha de Cronbach para las escalas utilizadas, son: valores, 0.75 ; creencias, 0.84; representaciones mentales, 0.83 . El instrumento aplicado tiene validez de contenido, pragmática, concurrentes, predictiva.

Características sociodemográfica. Los resultados indican las siguientes características: estrato uno $(9.9 \%)$, dos $(56.8 \%)$, tres $(28.4 \%)$ y cuatro $(5.1 \%)$; el nivel de educación correspondió a primaria (6.5\%), secundaria (44.1\%), universitario (48.3\%) y ninguno $(1.1 \%)$; la relación de parentesco fue solteros $(48.5 \%)$, en unión libre $(23.5 \%)$, casados $(21.5 \%)$, separados $(4.7 \%)$ y viudos $(1.8 \%)$; la edad estuvo entre 16 y $30(67.6 \%)$, entre 31 y $50(28.9 \%)$ y más de $50(3.6 \%) ; y$, los géneros 
participantes fueron masculino $(64.7 \%)$ y femenino (35.3\%).

Valores. Los motociclistas infractores poseen un conjunto de valores que han actuado de modo negativo en la seguridad y movilidad vial (ver cuadro 1).

Cuadro 1. Valores de los motociclistas infractores

\begin{tabular}{|c|c|c|}
\hline Valores & Descripción & Fragmentos de la entrevista \\
\hline Autonomía & $\begin{array}{l}\text { Refiere a la capacidad de } \\
\text { independencia o forma de gobierno } \\
\text { de sí mismo (Labédie, \& Amossé, } \\
\text { n.d.) }\end{array}$ & $\begin{array}{l}\text { "Mientras manejo, hablo por celular" }(\mathrm{C}, \mathrm{F})^{2} \text {. } \\
\text { "Me paso la cebra y el carril de los colectivos a altas } \\
\text { velocidades" (C, M). } \\
\text { "Me gusta la velocidad, el traguito y no usar casco y } \\
\text { chaleco" (C, M). }\end{array}$ \\
\hline Logro & $\begin{array}{l}\text { Utilidad, provecho o beneficio que } \\
\text { se obtiene una cosa (Valdés, n.d.) }\end{array}$ & $\begin{array}{l}\text { "Uso la moto como mototaxi, transporte familiar, } \\
\text { rebusque" (B, M). } \\
\text { "Me voy rápido para el trabajo para vender más, } \\
\text { vendo mis productos todo el día" }(\mathrm{B}, \mathrm{M}) \text {. }\end{array}$ \\
\hline Diversión & $\begin{array}{l}\text { Es el estado de gozo que tiene el ser } \\
\text { humano mediante diferentes tipos } \\
\text { de objetos o acciones (Weber, } \\
\text { 2001). }\end{array}$ & $\begin{array}{l}\text { "A veces me excedo en la velocidad porque me gusta } \\
\text { el riesgo (B, M). } \\
\text { "Pasarme los s emáforos en rojo y hacer piquecitos" } \\
(\mathrm{C}, \mathrm{M}) \text {. }\end{array}$ \\
\hline Religiosidad & $\begin{array}{l}\text { Hace referencia al culto espiritual } \\
\text { donde la persona encomienda su } \\
\text { vida y la de sus posesiones } \\
\text { (Jacques, 1999). }\end{array}$ & $\begin{array}{l}\text { "Pido protección a todos los santos" (B, M). } \\
\text { "La moto se la tengo encomendada al Divino Niño } \\
\text { Jesús" (C, F). }\end{array}$ \\
\hline Vanidad & $\begin{array}{l}\text { Consiste en valerse de diferentes } \\
\text { posesiones o actitudes para } \\
\text { alcanzar admiración (Saada- } \\
\text { Gendron, 2005). }\end{array}$ & $\begin{array}{l}\text { "A veces me da pena porque el casco es feo y me da } \\
\text { oso" (B, F). } \\
\text { "“No uso el casco porque me veo mal" (B, F). }\end{array}$ \\
\hline
\end{tabular}

Fuente. 50 Entrevistas a profundidad

Del anterior cuadro puede apreciarse de los motociclistas infractores el interés por la autodirección, estimulación y tradición, aspectos que para Maslow (1970) y Haire, Ghiselli, \& Porter (1996) corresponden a necesidades sociales de pertenencia, estimación y autorrealización con respecto a su grupo social. De ello se infiere el desconocimiento de la normatividad de tránsito y del respeto por los otros conductores y peatones. Esto conlleva a que comportamientos como hablar por celular, exceso de número de personas, andar sin casco, no respetar los carriles, pasar el semáforo rojo y realizar competencias, se conviertan en fenómenos naturalizados de la movilidad vial que restan importancia a comportamientos que sí respetan las normas. De un lado, algunos de los motociclistas justificaron su actuar por la falta de tiempo para dar cumplimiento a sus obligaciones, aspecto ya señalado por Xie \& Parker (2002) en su estudio; mientras que otros manifestaron un deseo fuerte por experimentar riesgos. Este último grupo considera que ciertos símbolos de tránsito como semáforos, reductores de velocidad, no adelantar vehículos, glorieta, no realizar giros prohibidos, entre otros son indicadores de riesgos y una manera de darse pertenencia y estimación con su grupo social. De manera que, los aumentos de accidentalidad tienen estrecha relación con los factores de tiempo y búsqueda de sensación de riesgos, de lo cual puede inferirse que ambos grupos de motociclistas poca importancia prestan a la autoridad de tránsito ya que esta no actúa de modo eficaz.

$\mathrm{Al}$ examinar los argumentos que justifican el comportamiento de los motociclistas infractores, se identifica un comportamiento consciente del riesgo vial y un desconocimiento de la autoridad, debido a que esta aplica de forma laxa las sanciones. Al no desempeñar la norma su efecto ejemplarizante, cada una de las infracciones como hablar por celular, número de personas, andar sin casco, no respetar los carriles, pasar el semáforo rojo y realizar piques pasan a convertirse en fenómenos naturalizados de la movilidad vial. Para evaluar el aporte de los valores encontrados se utilizó el Análisis de Componentes Principales (ACP) que mediante cinco (5) factores explican el $58.6 \%$ de ellos, y se caracterizan por: $\mathbf{F}_{\mathbf{1}} \mathbf{2} \mathbf{2 8 . 1 \%}$. Afanados. Realizan infracciones siempre como pasarse un semáforo en rojo, manejar rápido o en contravía, en zonas peatonales, con sobrecupo para evitar tantos viajes y hacer giros prohibidos por falta de tiempo. $F_{2} \cdot \mathbf{1 8 . 1 \%}$. Vanidosos. Denominados así porque evitan el uso responsable del caso por prestarle importancia a su apariencia. $\mathbf{F}_{3}$. $5.2 \%$. Arriesgado. Cometen infracciones como manejar por las noches sin luces y hablar por celular. $\mathrm{F}_{4} \cdot \mathbf{3 . 9} \%$. Retadores. Los conductores asumen una actitud retadora frente a situaciones de amonestación o riesgo (por ej. conducir a alta velocidad, realizar piques y manejar bajo efectos del alcohol). $\mathbf{F}_{5 .} 3.3 \%$. Religiosos. Siempre al salir de algún lugar piden protección a la Providencia para proteger sus bienes y su vida, tras llegar a su lugar de destino dan las gracias.

2 Las convenciones utilizadas hacen referencia a la identificación del estrato y el género de la persona entrevistada, de la siguiente manera: B para identificar el estrato bajo, C (estrato medio), A (estrato alto), F (género femenino) y $\mathrm{M}$ (género masculino). 
Esta caracterización de perfiles de los motociclistas infractores permite deducir que algunos de ellos perciben su infracción como leve, mientras otros como grave; la justificación de su percepción está relacionada con el estado de ánimo, cuya variación se debe a las preocupaciones laborales, familiares y sociales; este desequilibrio emocional perturba la organización de los grupos humanos debido a que el principal interés del sujeto consiste en solucionar sus preocupaciones. Como toda preocupación refleja una dedicación de tiempo sobre una actividad, cuya importancia está dada por su rol económico, social y personal; es notorio percibir de los motociclistas la importancia del tiempo, razón por la cual manifiestan a menudo una sensación de búsqueda por disminuir toda pérdida de este (Xie\& Parker, 2002). Esta reacción psicológica ha hecho cambios sustanciales sobre las dimensiones de autoridad, la concepción del yo y los modos de negociación (Hofstede, 2001).

Los motociclistas infractores desatienden la primera dimensión en vista de no encontrarse supervisados por la autoridad competente, si bien existe representaciones simbólicas de esta como las señales de tránsito (por ej. semáforos), las revisiones técnico mecánica, la licencia de tránsito, entre otras, contempladas en el Código Nacional de Tránsito Terrestre colombiano (Pineda, 2012), su papel no tiene el mismo efecto que la autoridad de tránsito representada en por los seres humanos.

Esto se debe a que la investidura institucional dada por el Gobierno colombiano a un grupo de personas para desempeñar la figura de autoridades de tránsito tiene un mayor reconocimiento para los motociclistas infractores porque ellas pueden juzgar penalmente su conducta; las representaciones simbólicas de las autoridades de tránsito, no; incluso, los demás actores de la movilidad como los peatones, conductores de vehículos y de bicicletas tampoco son reconocidos debido a que están desprovistos de la investidura de poder (Fernández \& Basabe, 2007).

Por otro lado, la concepción del yo es una construcción cultural sobre las identidades de las personas y su rol en la sociedad que para los motociclistas está muy definido por su género. La superioridad de los sexos que subyace en el comportamiento de ellos ha identificado como principales actores a los hombres, especialmente jóvenes, enlasinfracciones(OleaLunda, Nordfjærnb, \& Rundmoc, 2012), debido a que el legado cultural en los hombres siempre ha destacado valores como el riesgo y la autodeterminación (Hofstede, 2001); no obstante, es necesario precisar que la intensidad de los mismos depende de la edad, la educación, el estrato, la relación de parentesco y de género (Inglehart, 2000).

Creencias. Las creencias de un grupo de los motociclistas infractores estuvieron en relación a las actividades laborales, familiares y personales. Destacaron la moto como una posesión de gran importancia para sus vidas porque esta permite su autorrealización y les genera múltiples beneficios como dar trabajo (por ej. mototaxismo, mensajería), ahorrar tiempo y reducir la congestión; mientras otros insistieron en la sensación de riesgo y confianza que la moto genera para sus propósitos personales (por ej. transgredir la normas de tránsito, destacarse ante los miembros de su grupo y generar admiración). En este sentido el cuadro 2 presenta dichas creencias.

Cuadro 2. Creencias de los motociclistas infractores de la ciudad de Neiva

\begin{tabular}{|c|c|}
\hline Creencias & Fragmentos de la entrevista \\
\hline 1. La moto me da trabajo & $\begin{array}{l}\text { "Uso la moto para lo que se ofrezca: mototaxi, mandados, } \\
\text { domicilios" (C, M). }\end{array}$ \\
\hline 2. La moto me ahorra tiempo & $\begin{array}{l}\text { "Uno en la moto vuela y más cuando está con afán" (C, } \\
\text { F). }\end{array}$ \\
\hline 3.La moto reduce la congestión & $\begin{array}{l}\text { "En los trancones, uno avanza más rápido, pues uno no } \\
\text { ocupa mucho espacio" (B, F). }\end{array}$ \\
\hline $\begin{array}{l}\text { 4. La moto me hace ser una persona } \\
\text { arriesgada }\end{array}$ & $\begin{array}{l}\text { "Uno tiene una sensación de estar en las nubes, volando, } \\
\text { corriendo a toda" (B, M). }\end{array}$ \\
\hline $\begin{array}{l}\text { 5. Las reglas se hicieron para } \\
\text { romperse }\end{array}$ & $\begin{array}{l}\text { "A veces el ritmo de la ciudad lo obliga a uno a } \\
\text { desobedecer las normas, por ejemplo cuando hay un } \\
\text { trancón uno tiene que salirse como sea" (B, M). }\end{array}$ \\
\hline $\begin{array}{l}\text { 6. Por mucha beba no se pierde la } \\
\text { conciencia }\end{array}$ & $\begin{array}{l}\text { "Hay veces, pero poquitas. Yo no tomo mucho y trato de } \\
\text { cuidarme" (B, M). }\end{array}$ \\
\hline 7. El casco da caspa o infecciones & $\begin{array}{l}\text { "Por la calor le pica a uno mucho la cabeza y le da caspa" } \\
(\mathrm{B}, \mathrm{M}) \text {. }\end{array}$ \\
\hline $\begin{array}{l}\text { 8. El casco no me lo pongo porque es } \\
\text { feo }\end{array}$ & $\begin{array}{l}\text { "Es muy feo y uno no se siente bien manejando con } \\
\text { casco" (C, F). }\end{array}$ \\
\hline
\end{tabular}

Fuente: 50 Entrevistas a profundidad

De estas creencias se infiere que la primera de estas emergió debido al creciente desempleo. Ante esto, las personas buscan alternativas para solucionar las necesidades de su diario vivir (alimentación, pago de servicios, deudas, etc.); por esta razón, la moto es concebida como una forma ágil de proporcionar ingresos y aún más 
cuando se forma parte del sector informal. La segunda se origina a razón del ahorro de tiempo, el tamaño de la moto y su rapidez. Además, se deduce estos beneficios que aprovechados al máximo por los motociclistas si cuentan con muy buenas habilidades de conducción.

La tercera creencia está en relación con la anterior y es ideada de esta forma porque los motociclistas conciben que el tamaño de su moto constituya un factor decisivo en la movilidad vial, pues no ocupan tanto espacio como los automóviles y permiten que los retrasos de tiempo para el desarrollo de determinadas actividades sean mínimos. La cuarta creencia destaca los desafíos como una manera de autorrealización, de modo que la destreza para conducir como para evitar cualquier accidente a alta velocidad constituye una forma en que el motociclista caracteriza su identidad frente a su grupo de referencia.

La importancia que adquiere para los motociclistas su estatus lleva a que descuiden su responsabilidad social sobre la prevención de accidentes; de este modo, puede identificarse que la necesidad de admiración es un factor que regula el actuar de estos individuos. La quinta creencia surge para evitar dificultades en el diario vivir de las personas y están asociadas a situaciones de mucha importancia para la persona como llegar a tiempo al trabajo, al estudio, a una cita médica. Esto permite apreciar que gran parte de las justificaciones tienen como prioridad los aspectos personales y en menor importancia la seguridad vial. La sexta creencia surge de los incidentes de los motociclistas en las cuales han presenciado como espectadores o actores de los accidentes y manifiesta un grado de responsabilidad al no beber en exceso; aún así, ellos reinciden en el consumo de alcohol porque no perciben medidas restrictivas a su actuar. En ese sentido, podemos identificar un rol de la autoridad de tránsito condescendiente con dichas conductas infractoras, incluso cuando presencian espacios de celebración.

Laséptimayoctava creencia delosmotociclistas infractores se origina por la importancia que algunos de los motociclistas han dado a la vanidad, de modo que el uso de cualquier objeto está en relación a exaltar atributos de la persona y para otros motociclistas el interés está dirigido a la salud. De esta manera, la elección y uso del casco está en relacionado a estos dos aspectos. Así mismo, otros elementos de seguridad como el chaleco y los guantes, e incluso la moto, están siendo utilizados en un sentido estético y no preventivo de la seguridad vial.

Hemos presentado las razones dadas por los motociclistas para justificar las creencias sobre sus infracciones y todas presentan el componente emocional como regulador de la toma de decisiones de la persona, a tal punto de contrariar el sentido ético de sus derechos; de este modo, las creencias no manifiestan ninguna preocupación por la seguridad de los demás y esto ha hecho catalogar a los motociclistas como objeto de rechazo por la ciudadanía.

Para evaluar el aporte de las creencias encontradas entre los infractores se utilizó el Análisis de Componentes Principales (ACP) que mediante tres (3) factores explican el $60.8 \%$ de ellos, cuyas principales características son: $F_{1}$ (25.2\%). Imprudentes. Los motociclistas no cumplen con las normas de tránsito porque afectan el desarrollo normal de sus deberes laborales, familiares, sociales, por tal razón prefieren ignorarlas. $F_{2}$ (23.4\%). Vanidosos. Los motociclistas consideran que el casco, el chaleco y la moto deben darle una buena imagen que lo destaque ante los demás, si esta condición no es cumplida no son utilizados los implementos de seguridad. $F_{3}(\mathbf{1 2 . 2} \%)$. Fuente de trabajo. Los motociclistas utilizan la moto como fuente de trabajo porque carece de un empleo, y por la facilidad de este para cumplir con las tareas laborales es poco frecuente la evasión de la normatividad para mantenerse en aquél.

Estos sistemas de creencias hallados no contienen formas irracionales, sino una organización racional donde los motociclistas han catalogado las creencias en función de la actividad desempeñada con más frecuencia. Tres condiciones permiten esta organización racional de la creencia: la proximidad referida a la relación cognoscente entre el sujeto y un objeto, 
situación o personas para poder generarse. Una segunda condición alude a la valoración, esta hace referencia a la importancia de una creencia entre sus miembros y su entendimiento es con base al contexto del cual emerge. Una última condición de conformación es la sensación de control sobre el objeto (Bergner \& Ramon, 2013).

La primera condición da cuenta de que los conductores infractores reconocen las oportunidades para cometer la infracción; la existencia de la infracción, como señalamos en el acápite de valores, obedece a la falta de interiorización de la normatividad; por tanto, la debilidad institucional de las autoridades de tránsito está conllevando a las personas a aceptar las infracciones como parte natural del proceso de movilidad vial. De este modo, la segunda condición revela que las autoridades de tránsito no hacen uso plenamente de sus facultades para el cumplimiento de la norma, ya que su decisión está condicionada a situaciones como ausencia de cargo, soborno o relaciones de poder. De ahí concluimos que la tercera condición facilita a los motociclistas infractores considerarse como el actor principal en la movilidad vial al cual los demás actores (conductores, peatones, ciclistas) deben someterse.

Sin embargo, llama la atención como tales creencias sobre las infracciones pierden su existencia en el momento que la autoridad de tránsito forma parte del contexto de la movilidad vial, afectando las intenciones de los motociclistas sobre la realización de la infracción; esto ocurre por la vestimenta institucional, la cual le otorga un poder a la autoridad para obrar sobre la otra persona cuando la última realiza acciones en contra del bienestar de los demás; tales acciones son mecanismos de adecuación conductual. Vale la pena aclarar, los mecanismos no eliminan el comportamiento indeseable, sino que sensibilizan a la persona para no reincidir en acciones contrarias a la norma.

De ahí que en todo lugar existan indicadores conductuales como señales, gestos, comunicaciones, etc. cuya función es recordarle a la persona su responsabilidad en la sostenibilidad del bienestar social, particularmente, en la movilidad vial. En ese sentido, pudo identificarse dentro de los motociclistas infractores que algunos $(59,7 \%)$ reinciden bastante en las infracciones de mayor impacto en la movilidad vial como son: conducir a alta velocidad, pasarse los semáforos en rojo, conducir con sobrecupo; mientras otros $(40,3 \%)$ realizan infracciones de impacto mediano como hablar por celular, distraerse, no utilizar casco ni chaleco. En ambas situaciones se revela la debilidad institucional de la autoridad de tránsito para dar cumplimiento a la norma.

Representaciones sociales. Las representaciones sociales de los motociclistas infractores dan cuenta que la moto puede ser concebida de tres maneras a saber: como un medio para una oportunidad laboral, como vehículo práctico por su rapidez y tamaño cuyo aspecto destacaron autores como Nallet, Bernard \& Chiron (2008); así mismo, los accesorios pueden ser considerados como posesiones que fortalecen el sentido estético. A continuación se relacionan dichas representaciones (ver cuadro 3 ).

Cuadro 3. Representaciones sociales de los valores de los motociclistas infractores de la ciudad de Neiva.

\begin{tabular}{c|l}
\hline Representación social & \multicolumn{1}{c}{ Fragmentos de la entrevista } \\
\hline $\begin{array}{c}\text { 1. Las reglas se hicieron } \\
\text { para romperse. }\end{array}$ & $\begin{array}{l}\text { "Tengo que estar en la jugada y pues con la congestión vial uno debe } \\
\text { adelantarse en el carril andando rápido" (C, M). }\end{array}$ \\
"Yo me tomo unas polas y luego me voy para la casa en la moto, \\
igual como uno sabe en dónde están los guarda se va por otro lado" \\
(C, M).
\end{tabular}

Fuente: 50 Entrevistas a profundidad

De este cuadro podemos identificar que en la primera representación, además de las infracciones mencionadas por los motociclistas infractores, estos manifestaron actitudes agresivas cuando los otros conductores impiden las infracciones. De manera que los motociclistas infractores son considerados por otros motociclistas, conductores de carro, ciclistas y peatones, como un grupo de persona riesgosas; este aspectoestá en relación con el estudio de Ruiz, Gómez, Beltrán, Lamus \& Leal (2014) acerca de los efectos de la agresividad y ansiedad sobre la conducción y la accidentalidad. Contrariamente, 
un número menor de motociclistas infractores son considerados de menor peligrosidad vial porque tienen en cuenta la seguridad de la otra persona, previendo antes de realizar la infracción que no haya ninguna persona $o$ conductor expuesto. De lo anterior si infiere que estos motociclistas infractores asumen que la preocupación por los demás reduce la censura a su comportamiento infractor y el número de accidentes.

La segunda representación se condensan otros aspectos que dan origen a esta, a saber: una mayor panorámica de la vía, un tamaño menor -atributo señalado por Cantin, Lavallière, Simoneau \& Teasdale (2009) en su estudio-, cilindraje y ahorro del consumo de gasolina; de ahí que predomine una representación social de la motocicleta como vehículo práctico, esta representación social no es equivalente para los conductores de carros, quienes consideran la moto como un vehículo inseguro (Walker, Stantonb \& Salmonc, 2011), más cuando se emplea para actividades laborales como mototaxismo, mensajería, etc.

Finalmente, la tercera representación da cuenta que algunos de los motociclistas infractores consideran que su imagen personal es importante ya que les permite ser parte de otros grupos sociales; de esta manera, los motociclistas han optado por evitar el uso del casco e incluso el chaleco, aunque según pudo apreciarse de las opiniones de los entrevistados el uso del casco, y en raras ocasiones el chaleco, ocurre cuando estos observan la presencia de una autoridad. Para evaluar el aporte de las representaciones sociales encontradas entre los infractores se utilizó el Análisis de Componentes Principales (ACP) que mediante tres (3) factores explican el $60.8 \%$ de estas, encontrándose la

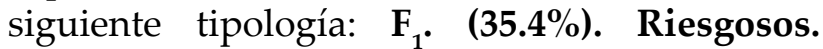
Los motociclistas infractores conciben la moto como un medio que les permite autoafirmar su autonomía, debido a que el conductor tiene la sensación de control mientras conduce y para no perderla realiza acciones contrarias a la norma. $F_{2}$. (16.2\%). Trabajadores. Los motociclistas infractores infringen la norma para cumplir con los deberes de sus empleos, particularmente en sector informal, por ej. mototaxismo, mensajería, entre otras. $\mathbf{F}_{3}$. $\mathbf{9 . 1 \% )}$. Rebeldes. Los motociclistas infractores no encuentra utilidad en el casco, por eso evitan usarlo. Además, justifican su inutilidad por razones estéticas y climáticas.

De lo anterior puede identificarse que sigue fortaleciéndose la concepción de una movilidad vial sin control normativo $\mathrm{y}$ presencia de las autoridades, ante la cual las infracciones siguen en aumento especialmente en poblaciones de género masculino y con edades menores a los treinta años (González \& Ossa, 2011): Como podemos identificar en la representación social de riesgosos, y con base en el perfil sociodemográfico de los motociclistas infractores, la población joven en edad de 16 a 30 años tiene gran participación en esta representación (67.6\%), y en menor medida aquellas en edades entre 31 y $50(28.9 \%)$ y más de $50(3.6 \%)$ distribuidas entre los trabajadores y rebeldes. Otro aspecto deducido de estas representaciones es la relación con los demás conductores donde existe una fuerte presencia de violencia de los motociclistas infractores hacia otros actores de la movilidad vial (Ruiz, Gómez, Beltrán, Lamus, \& Leal, 2014): un gran porcentaje de los motociclistas infractores son considerados riesgosos porque desarrollan actitudes de intolerancia y agresión y efectúan infracciones de un gran riesgo como conducir a alta velocidad o manejar bajo estado de alicoramiento.

Contrariamente un número menor de motociclistas infractores son considerados de menor peligrosidad vial porque en apariencia tienen en cuenta la seguridad de la otra persona, previendo antes de realizar la infracción que no haya ninguna persona o conductor expuesto. Algunas situaciones son pasarse el semáforo en rojo, hacer giros prohibidos, hablar por celular, no usar casco, entre otras. En ese sentido, este grupo de motociclistas infractores asumen que la preocupación por los demás reduce la actitud orientada al riesgo y causalidad de accidentes como también reduce la censura a su comportamiento infractor (Cantin, Lavallière, Simoneau, \& Teasdale, 2009). 
Aunque las infracciones de tránsito cometidas están catalogadas de acuerdo a los efectos sociales y penales, podemos identificar que las representaciones sociales de los motociclistas infractores dan cuenta de una norma subjetiva ${ }^{3}$, la cual puede estar fundamentada por falsas ideas (la inexistencia de la norma, la condescendencia de los conductores, entre otras) como está ocurriendo con los motociclistas infractores. Ellos asumen las infracciones como parte de su vida y hacen extensiva aquellas en espacios con una función diferente como las zonas residenciales y los espacios públicos (Jodelet, 2006). Pese a las oposiciones hechas por la ciudadanía sobre tal conducta, la herencia social entre los mismos motociclistas e incluso otros conductores de vehículos ha desfavorecido la figura del ciudadano a falta de una regulación normativa más satisfactoria (Rateau \& Monaco, 2013).

En ese sentido, al reflexionar los motociclistas infractores sobre su imagen social, mediante el Análisis de Clúster, se encontraron tres grupos, cuyos imaginarios sobre su comportamiento se caracterizan por: $\mathrm{Cl}_{1} \cdot \mathbf{3 6 . 9 \%}$. Riesgosos. $\mathrm{La}$ ciudadanía y demás actores de la movilidad cuando piensan en ellos solo expresan inseguridad, accidentalidad $\mathrm{y}$ violencia. $\mathbf{C l}_{2}$. $35.3 \%$. Trabajadores. La ciudadanía y demás actores cuando piensan en estos motociclistas si bien reconocen la falta de oportunidades laborales no justifican su irresponsabilidad vial. $\mathbf{C l}_{3}$. $\mathbf{2 7 . 8} \%$. Rebeldes. La ciudadanía y demás actores cuando piensan en este tipo de motociclistas identifican que su conducta es resultado de una necesidad de autoafirmación con su grupo de referencia.

Cada uno de estos tipos de motociclistas infractores conciben idealmente que la movilidad vial es una acción y medio orientada a facilitar el tránsito de los vehículos; sin embargo, cuando dicha movilidad es afectada por el flujo de otros vehículos o peatones, los motociclistas infractores reprochan todo tipo de obstrucción que afecte su propia movilidad, pero no toleran cuestionamiento sobre ellos mismos cuando cometen la infracción. Esta forma de reaccionar obedece a que los motociclistas infractores consideran que sus asuntos personales tienen más importancia que los asuntos de movilidad y seguridad vial (Cantin, Lavallière, Simoneau, \& Teasdale, 2009), especialmente porque los asuntos de estas personas están en relación con temas como ansiedad, depresión, baja autoestima, entre otros (Cegarra, 2012).

\section{Consideraciones finales}

Los hallazgos indican que las infracciones son asumidas por los motociclistas infractores como parte de su vida y las hacen extensiva en espacios que poseen una función diferente como son las zonas residenciales y los espacios públicos. Sin embargo, la motivación entre los motociclistas para realizar las infracciones es diferente: un grupo de motociclistas están afectados por la noción de tiempo, pues entienden que las actividades que desempeñan adquieren un valor económico y social que les permite auto realizarse $\mathrm{y}$ considerarse perteneciente de su grupo social. De ahí que, la ansiedad e impulsividad sean características comunes a este grupo (Bachoo, Bhagwanjee \& Govender, 2013). El otro grupo está orientado a definir su identidad de acuerdo a la relevancia del grupo social en aspectos de posicionamiento cultural y no económico como se pensaría con el anterior grupo; este podría deberse a la "superioridad" entre los sexos señalado por Olea, Nordfjærnb \& Rundmoc (2012) ya que estos motociclistas, principalmente hombres jovenes, han sido receptores de una cultura masculina donde el riesgo se ha considerad o fuente de éxito personal, autonomía y poder. Lo anterior permite ratificar la presencia de los valores culturales como guías del comportamiento (Schwartz, 1994).

Incluso, el anterior planteamiento refuerza la interpretación de Hofstede (2001) sobre la

3 Jodelet (2006) indicó que el ser humano por su permanente interacción con los otros comparte significados sobre cómo comportarse, los cuales se constituyen en una presión social. Sin embargo, la interiorización de aquellos depende del vínculo emocional dado por la persona. 
incidencia de los valores en la conformación de otros sistemas de organización como por ejemplo las creencias y las representaciones sociales. Una primera razón refiere al reconocimiento de las propiedades de uno o varios valores en las actividades que desempeñan los motociclistas, sean estos infractores o no; de modo que estos valores les orienta el quehacer diario de sus vidas y la relación con los demás.

Una segunda razón, las creencias y representaciones sociales como formas de entendimiento de los motociclistas necesitaron de los valores para discenir sus experiencias dado que las creencias y representaciones sociales, además de utilizarlos para justificar sus conductas, establecieron sus carácteristicas de la siguiente manera: las creencias elaboradas por los motociclistas infractores se pueden entender solo en las situaciones que participan como el trabajo, la sensación de riesgo, por citar dos ejemplos. Mientras que las representaciones sociales se hacen extensivas a otros grupos sociales (conductores de carros, peatones, ciclistas) en tanto que los motociclistas dan un conjunto de imágenes acerca la manera como estos grupos, suponen ellos, los conciben.

Por otro lado, la distinción de los valores de los motociclistas infractores y los valores de los motociclistas no infractores, estos últimos orientados a la seguridad vial, nos permite aducir que el planteamiento de Inglehart (2000) sobre los valores como entes susceptibles de sufrir cambios es válido dado que la debilidad de las autoridades de tránsito para fortalecer dichos valores orientados a la seguridad vial tiene como dificultades el control poco riguroso del proceso de expedición de la licencia de tránsito (Ozkan, Lajunen, Chliaoutakis, Parker \& Summala, 2006) y un papel poco eficiente de las autoridades de tránsito en la aplicación de las sanciones correspondientes a la infracción. Para ilustrar lo anterior, existe tres formas de negociar de los motociclistas infractores: el diálogo, el soborno y la intimidación. El 42.3\% señaló emplear el diálogo aduciendo que pocas veces incumplen las normas de tránsito y aceptan su responsabilidad y sin problema el comparendo. Por el contrario, un $30.2 \%$ aunque reconoce su equivocación, ofrece dádivas al policía de tránsito o a la persona afectada a cambio de su silencio; $\mathrm{y}$ un $21.2 \%$ reconoce utilizar las relaciones interpersonales de poder (conoce autoridades de tránsito, labora en organizaciones públicas) para intimidar y evitar la sanción.

De esta manera los símbolos de seguridad vial como las señales de tránsito, las revisiones técnico mecánicas, el uso del casco, entre otras, contempladas en el Código Nacional de Tránsito Terrestre colombiano (Pineda, 2012) han pasado de considerarse como un deber a una situación de elección. Esto conlleva a que actores como los conductores de carro, los ciclistas y los peatones estén condicionados a las decisiones de los infractores (Bergner \& Ramon, 2013). Incluso, los lleva a desarrollar mecanismos alternos (ademanes, gritos) para recordarle al infractor su responsabilidad en la seguridad y movilidad vial.

\section{Referencias}

Bachoo, S., Bhagwanjee, A., \& Govender, K. (2013). The influence of anger, impulsivity, sensation seeking and driver attitudes on risky driving behaviour among postgraduate university students in Durban, South Africa. Accident Analysis and Prevention (55), 67-76.

Bergner, R. M., \& Ramon, A. (2013). Some Implications of Beliefs in altruism, free Will, and nonreductionism. The Journal of Social Psychology, 153(5), 598-618.

Cantin, V., Lavallière, M., Simoneau, M., \& Teasdale, N. (2009). Mental workload when driving in a simulator: effects of age and driving complexity. Accident Analysis $\mathcal{E}$ Prevention, 41(4), 763-771.

Garfinkel, H. (1967). Studies in Ethnomethodology. Englewood Clifs: Prentice Hall.

Gaygisiz, E. (2010). Cultural values and governance quality as correlates of road traffic fatalities: A nation level analysis. Accident Analysis and Prevention (42), 18941901. 
Gomes Vieira, Sandra. (2013). The influence of the infrastructure characteristics in urban road accidents occurrence. Accident Analysis and Prevention, 60, 289-297.

González G., J., \& Ossa N., P. (2011). La movilidad de los motociclistas en la ciudad de Neiva. Conocimiento UH, 3(2), 21-27.

Hofstede, G. (2001). Cultura y organizaciones. Madrid: Alianza Editorial.

Inglehart, R. Y. (2000). Modernization, cultural change, and the persistence of traditional values. American Sociological Review (1), 1951.

Jacques, R. (1999). Le "spirituel" et le "religieux" à l'épreuve de la transcendance. Théologiques, 7(1), 89-106.

Labédie, A., \& Amossé, G. (n.d.). Constructivisme et socio-constructivisme. Recuperado el 3 de Mayo de 2015, de http://portail-du-fle.info/ glossaire/Constructivisme.html

Maslow, A. H. (1970). Religions, values, and peak experiences. New york: Penguin.

Nallet, N., Bernard, M., \& Chiron, M. (2008). Individuals taking a French driving licence points recovery course: Their attitudes towards violations. Accident Analysis and Prevention (40), 1836-1843.

Naveteur, Janick; Coeugnet, Stéphanie; Charron, Camilo; Dorn, Lisa; Anceaux, fançoise. (2013). Impatience and time pressure: Subjective reactions of drivers in situations forcing them to stop their car in the road. Transportation Research Part F: Traffic Psychology and Behaviour, 18, 58-71.

Olea Lunda, I., Nordfjærnb, \& Rundmoc, T. (2012). Associations between risk judgments and demand for transport. Journal of Risk Research, 1-12.

Ozkan, T., Lajunen, T., Chliaoutakis, J., Parker, D., \& Summala, H. (2006). Cross-cultural differences in driving behaviours:A comparison of six countries. Transportation Research Part F (9), 227-242.

Pineda G., O. D. . (2012). Régimen Jurídico del tránsito. Recuperado el 15 de Abril de 2015, de http://www.fpv.org.co/uploads/ repositorio/regimen_juridico_del_ transito_terrestre_colombia_2012.pdf

Poncela F., A. (2012). Representaciones sociales: mujeres al volante ¿peligro constante? Iberofórum.Revista de Ciencias Sociales de la Universidad Iberoamericana (14), 1-34.

Ruiz P., J. I., Gómez, I. A., .Beltrán, I. T., Lamus, D. A., \& Leal S, L. J. (2014). Representaciones sociales de normas de tránsito, agresividad, facilidad percibida en la conducción, accidentes y multas en conductores de Bogotá, D. C. Revista Criminalidad, 56(2), 291-308.

Saada-Gendron, J. (2005). L'analyse des passions dans la dissolution du corps politique: Spinoza et Hobbes. Recuperado el 16 de Enero de 2015, de http://asterion.revues.org/157?lang=en

Schwartz, S. (1994). Are there universal aspects in the structure and content of human values? Journal of Sociology(50), 19-45.

Strauss, A., \& Corbin, J. . (1998). Bases de la Investigación Cualitativa. Antioquia: Editorial Universidad de Antioquia.

Valdés de la H., R. . (n.d.). El interés jurídico procesal. Recuperado el 3 de Mayo de 2015, de http://www.letrasjuridicas.com/ Volumenes/8/delahuerta8.pdf

Vargas Castillo, D. A. (2013). Comportamiento de muertes y lesiones por accidente de transporte, Colombia. Recuperado el 20 de Abril de 2015, de http://www.medicinalegal. gov.co/documents/10180/188820/ F O R E N S I S + $2013+4-$ +accidentes+de+transporte.pdf/51867e309ab5-4a15-8363-f2232d2c86ae 
ENTORNOS, Vol. 29, No. 1, Junio 2016

Walker, H., Stantonb, N., \& Salmonc, P. (2011). Cognitive compatibility of motorcyclists and car drivers. Accident Analysis and Prevention (43), 878-888.

Weber, D. (2001. (2001). La fuite de soi, le masque du divertissement. Études, 631-641.

Xie, C., \& Parker, D. (2002). A social psychological approach to driving violations in two Chinese cities. Transportation Research Part F, 293-308. 